\title{
Statistical Machine Translation Outperforms Neural Machine Translation in Software Engineering: Why and How
}

\author{
Hung Phan \\ Iowa State University \\ Ames, USA \\ hungphd@iastate.edu
}

\author{
Ali Jannesari \\ Iowa State University \\ Ames, USA \\ jannesari@iastate.edu
}

\begin{abstract}
Neural Machine Translation (NMT) is the current trend approach in Natural Language Processing (NLP) to solve the problem of automatically inferring the content of target language given the source language. The ability of NMT is to learn deep knowledge inside languages by deep learning approaches. However, prior works show that NMT has its own drawbacks in NLP and in some research problems of Software Engineering (SE). In this work, we provide a hypothesis that SE corpus has inherent characteristics that NMT will confront challenges compared to the state-of-the-art translation engine based on Statistical Machine Translation. We introduce a problem which is significant in $\mathrm{SE}$ and has characteristics that challenges the ability of NMT to learn correct sequences, called Prefix Mapping. We implement and optimize the original SMT and NMT to mitigate those challenges. By the evaluation, we show that SMT outperforms NMT for this research problem, which provides potential directions to optimize the current NMT engines for specific classes of parallel corpus. By achieving the accuracy from $65 \%$ to $90 \%$ for code tokens generation of 1000 Github code corpus, we show the potential of using MT for code completion at token level.
\end{abstract}

\section{CCS CONCEPTS}

- Software and its engineering $\rightarrow$ Source code generation.

\section{KEYWORDS}

Neural Machine Translation, Statistical Machine Translation

\section{ACM Reference Format:}

Hung Phan and Ali Jannesari. 2020. Statistical Machine Translation Outperforms Neural Machine Translation in Software Engineering: Why and How. In Proceedings of the 1st ACM SIGSOFT International Workshop on Representation Learning for Software Engineering and Program Languages (RL+SE\&PL '20), November 13, 2020, Virtual, USA. ACM, New York, NY, USA, 10 pages. https://doi.org/10.1145/3416506.3423576

\section{INTRODUCTION}

Deep Learning (DL) has been applied in different Software Engineering (SE) researches and problems[6, 12, 27]. It can contribute to all stages of software development life cycle, from requirements extraction, design, implementation to maintenance [27]. Since DL

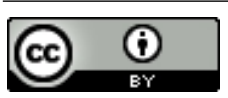

This work is licensed under a Creative Commons Attribution International 4.0 License.

$R L+S E \& P L$ '20, November 13, 2020, Virtual, USA

(C) 2020 Copyright held by the owner/author(s).

ACM ISBN 978-1-4503-8125-3/20/11.

https://doi.org/10.1145/3416506.3423576 was vastly applied earlier in Natural Language Processing (NLP), a popular trend of applying DL in SE is to consider the input of SE problems as different types of documentation similar to NLP input. According to [6], there are 3 main types of documentation: Natural Language (NL), Software Documentation (SD) and Programming Language (PL). Based on the requirement of each tasks, the output of research works that used these types of documentation as input is varied by different types of code tokens. For the research works that used NL as input, they tend to find the element of code environment that satisfied the description in NL, which they applied for code search $[4,15]$ and code synthesis [28]. SD is special type of documentation written in NL but contains information about description of the Application Programming Interfaces (APIs) of different programming languages. There is a work in the literature on representing APIs as vector from SD [45]. For the research works used PL as input, they used deep learning translation between PLs [11] and code suggestion [10].

The output of DL researches in SE problems can not only be the source code or code tokens, but also could be the information from SDs and NLs. Different applications are proposed to translate between each types of documentations using Machine Learning (ML). There are works on generating SD as pseudo-code from code using DL and ML $[2,33]$, or generating documentation from API specifications [36], or generating commit messages in NL [29]. Neural Machine Translation (NMT), which is a technique relied on advantages of DL, can be assumed as the best translation engine for SE. The ability of NMT relies on the formation on multiple layers of neural network to capture more information for the translation of each elements in the source language [48]. Besides, along with text sequence, NMT can be applicable on a different data structure such as graph or tree, which is suitable for the representation of code [47]. Another advantage of NMT is the performance for inferring the results, which is usually outperform earlier Machine Translation techniques [48].

Before the era of NMT, Statistical Machine Translation (SMT) [9] was the most popular technique for solving SE problems which relied on MT approaches. With the idea of extending the original Bayes rule [44], SMT provides the ability of learning the context in Natural Language for translation between popular languages. Since the source code also embed information of NL [19], SMT is successfully be applicable of SE problems as translation between versions of Python [23] and between different PLs [32]. However, compared to newer trends of translation engines such as NMT, SMT reveals 2 drawbacks. First, it cannot learn information from long sequence of text. An implementation tool of SMT, Phrasal [14] can only process the phrase with maximum length of 7 . Secondly, the training and testing time of SMT become worse with large training 
data and increases exponentially [41]. For these reasons, NMT has replaced SMT in SE problems [2,33].

Although having many advantages, NMT itself contains some challenges which also appear in researches of different areas along with SE. [31, 46] mention about an important problem of NMT compared to SMT as rare words problem in NLP. Current popular NMT engines, OpenNMT [25] or Google NMT [30], cannot handle large size vocabulary with more than 100000 words. To optimize the problem, researchers considered rare words as Unknown words which their translated results are not counted to the final results. This fact caused NMT performs poorly when rare/unknown words are frequent in the corpus [31]. In SE researches, the problem of Type Inference using MT shows that SMT model provided by [37] has a significant higher accuracy compared to the original NMT approach in [18]. Similarly, for natural language diacritic restoration, [35] shows that SMT outperforms NMT. [18, 30, 37] have the same characteristics of parallel corpus, i.e., the length of source and target pairs are equal and the order of the source and target words are consistent with each other. This leads us to an assumption that if the parallel corpus for training MT has these characteristics, it will affect the accuracy of NMT. In summary, NMT tends to have lower accuracy than SMT due to the methodology of NMT that didn't support the rare words translation and the characteristics of parallel corpus.

In this work, we further investigate the efficiency of NMT vs. SMT in a new research problem that has similar characteristics of parallel corpus as [18, 30, 37]. Instead of focusing on only limited types of tokens in code environment, our problem provide a solution to help developers get the code of all types of code tokens based on its first letters in the form of abbreviation/prefix of tokens. To implement the solution for this problem, we build two spaces of abbreviations of code tokens as source language and code tokens as target language. Then, we implement two machine translation models, Neural Machine Translation and Statistical Machine Translation to learn the mapping from prefixes to code tokens for code suggestion. We also analyze the affect of unknown tokens along with the accuracy on each types of tokens. By the evaluation, we show that SMT outperforms the original NMT. We called our approach PrefixMap and analyze the effective translation on three types of documentation: NL, SD and PL. Overall, this paper provides the following contributions:

(1) Proposing the translation based engine for code completion from first letters of tokens.

(2) Providing algorithms for extracting parallel corpus of prefixes and tokens in 3 types of documentation used in NLP and SE.

(3) Implementing and Optimizing Statistical Machine Translation for PrefixMapping.

(4) Implementing and Optimizing Neural Machine Translation for PrefixMapping.

(5) Analyzing the accuracy of NMT compared to SMT along with accuracy depending on each types of code tokens.

The structure of the paper is provided as follow. In the next section, we will describe about our research problem. In this section, we will define important concepts we use for abbreviations, algorithms for data collection, and the overview architecture of the
Table 1: Example of source target tokens for Prefix Mapping at n-letter(s) prefixes

\begin{tabular}{|c|c|c|}
\hline \multirow{3}{*}{ Source } & $\begin{array}{l}\text { 1-letter } \\
\text { prefix }\end{array}$ & 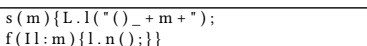 \\
\hline & $\begin{array}{l}5 \text {-letters } \\
\text { prefix }\end{array}$ & $\begin{array}{l}\text { synch (mList) }\{\text { LogUt } \\
. \log W(\text { "Regu ( ) list }+\ldots\end{array}$ \\
\hline & $\begin{array}{l}\text { 9-letters } \\
\text { prefix }\end{array}$ & $\begin{array}{l}\text { synchroni }(\text { mListener })\{\text { LogUtils . } \log W \\
\left(\text { "Request } Q() \_ \text {listener }+\ldots\right.\end{array}$ \\
\hline Target & $\begin{array}{l}\text { Code } \\
\text { tokens }\end{array}$ & $\begin{array}{l}\text { synchronized ( mListeners) \{ LogUtils. } \log W \\
\text { ("RequestQueue.notifyOfItemInRequestQueue ( )... }\end{array}$ \\
\hline
\end{tabular}

system. The core engines of translation, NMT and SMT, along with their optimization for this problem is described in the third section. In the evaluation, we will provide the accuracy and head-to-head comparison between NMT and SMT in 3 types of documentation.

\section{PREFIX RESOLUTION}

$[18,37]$ provide a translation approach considered the source side language as partial class name (PCN) and the target language as Fully Qualified Name (FQN) of APIs. [35] treated the source language as a word without diacritic information and the target language as a word with diacritic information. In other words, both of these research works build a parallel corpus with the same length of source sequence and target sequence to each pair. The orders for source and for target sequences are also consistent. In summary, the problem of translation in $[18,35,37]$ has the common in characteristics of parallel corpus.

In our work, we design an inferring system based on translation inspiring from [37]. While [37] focuses on the class name of APIs as the source language, we provide our source language as types of abbreviation for each words in a documentation corpus. Depending on different level of abbreviation, we build multiple translation models based on different length of the first letters for each words in parallel corpus. We have some definitions of elements in our context of translation problem.

Definition 2.1. Prefix: Given a word or code token, the prefix of a word is a word that combined by the set of first letters which developer can input to the code editor.

Definition 2.2. n-letter(s) Prefix: The n-letter prefix of a word/ code token is the prefix that has length of $\mathrm{n}$ letter.

We see examples of these definitions in PL in the Listing 1. This code snippet is the method declaration of event function fireQueueStateChanged() from [7]. This function is to handle the event for Android version of Vodaphone devices. From this function, we see that there are many kinds of code tokens, including program keywords, class name, method name and variable names. Look at the token notifyOfItemInRequestQueue() as an example. In this token, its 1-letter prefix is n. Its 3-letters prefix is not and 9-levels prefix is notifyOfI. We provide a code suggestion that allows developers to write multiple prefixes at 1 level, 3 levels and 9 levels. Then, in the next step, the machine translation will translate from the area of n-levels prefixes to suggest the full tokens. We do not restrict on any kinds of code tokens. In the other words, we support suggesting tokens for all types of prefixes.

To implement the solution, we do the following steps. First, we collect the data for software projects. Next, we extract the information from source as prefixes and target as the code tokens by the 
visiting code at Abstract Syntax Tree (AST) tree structure. Depending on the how many letters of the input prefix, the application will load the training models for the same letters prefixes inference. In the final step, the suggestion of code tokens from prefixes is provided. Example of tokens from source and target language at different $n$-letters is provided by Table 1 .

\section{Listing 1: Example of code snippet [7]}

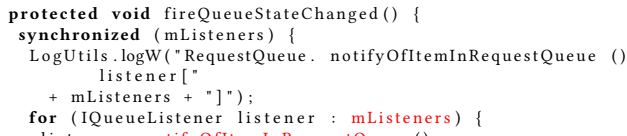

\subsection{Architecture Overview}

The architecture overview of our tool, PrefixMap is provided as follows. We provide a code editor that accept developers to write abbreviation of code tokens in the form of first letters. After this step, we have the input as the mix of full code tokens and prefixes. The length of prefix can be varied. Then the information of code will be parsed by an Abstract Syntax Tree (AST) Parser to collect the code to sequence of prefixes. Each prefixes of code tokens will be encoded into the sequence as the source sequence for translation. Then, the MT engines will convert the sequence of prefixes to sequence of tokens, which shows suggestions for each input prefixes. For example, $\mathrm{mList}$ and notif prefixes in Listing 1 will be translated to the expected tokens which are $\mathrm{mListeners}$ and method name notifyofItemInRequestQueue. To be able for inference in the testing phase, we provide the training phase with the source language as prefixes and target language as code tokens from 1000 Github Corpus we collect from MSR 2013 [3].

From the architecture overview, we can see two important points we need to address compared to other neural machine translation works. First, we considered the both 2 MT engines for fixing the prefixes. We show the strength and the disadvantages of each MT models for this problem. Secondly, the PrefixMap was trained based on the parallel corpus with consistent in length of tokens and order of source and target tokens. Third, in our approach, each source tokens needs to be mapped with a target tokens, means the cases of unknown tokens will be affected since the MT cannot provide the suggestion. We will study about the affect in the Evaluation section.

\section{MACHINE TRANSLATION ENGINES}

In this section, we discuss about the algorithm for data extraction and the algorithm for SMT and NMT.

\subsection{Data Extraction}

The algorithm of extracting source side and language side is provided in Listing 2. The core of implementation is done by an AST parser we extended from the source code of Eclipse JDT [20]. In this parser, we enhance the default visit() function which accepts an ASTNode object to a new function visitAndExtract(). This function behaves specifically to the MethodDeclaration node and the ASTNode objects inside this MethodDeclaration. The function for other AST objects accepts the node, the level of prefixes and the pair object. For each pairs, they contain one sequence of tokens as source and one sequence of tokens as target. Inside this function, first it will extract all tokens inside the ASTNode. Next, it will extract the prefix at n-levels for each tokens by a for loop. Final, the source and target sequence are included in the a new Pair object before adding the new object to the list. The visitAndExtract() function for MethodDeclaration accepts a list of pairs as input. It will create a new Pair object, visiting each ASTNode objects in the sub tree of this MethodDeclaration and extract the prefixes and related code tokens in training Github projects.

\section{Listing 2: Algorithm to extract sequences in Pre- fixMap}

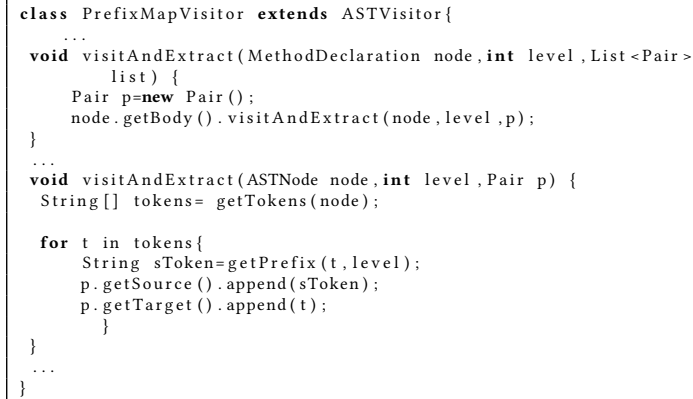

For example in Listing 1, the algorithm Listing 2 will visit the fireQueueStateChanged() MethodDeclaration to extract information all ASTNode inside this function. The information provides us the content of varies of types of ASTNode, including the MethodInvocation, for loop and inside variables and inside MethodInvocation. The target side for each tokens is actually the code content, while the source side contains the prefix of the code tokens, which can be inputted by developers in the suggestion phase. There is a corner cases that the size of the required prefix is greater than the length of the code tokens. If that case happened, the prefix will encode the information of the whole token. For instance, the token for has the prefix at 9-letters as for. In the next section, we will discuss about important elements of MT engines we used.

\subsection{Statistical Machine Translation for Prefix Map}

Our implementation of SMT for PrefixMap is based on a well-known toolkit Phrasal [14] from StanfordNLP group. We call the source sequence as Prefixes and the target tokens as Codes. We call pre fix $i$ as the $i^{t h}$ index prefix in the source sequence and code $i$ as the $i^{t h}$ index token in the target sequence. The purpose of SMT, along with NMT, is to calculate probability of each prefix is translated to target token given a context of code by different machine learning directions. For SMT, this probability contains 2 elements : the Language Model (LM) and Translation Model (TM), 


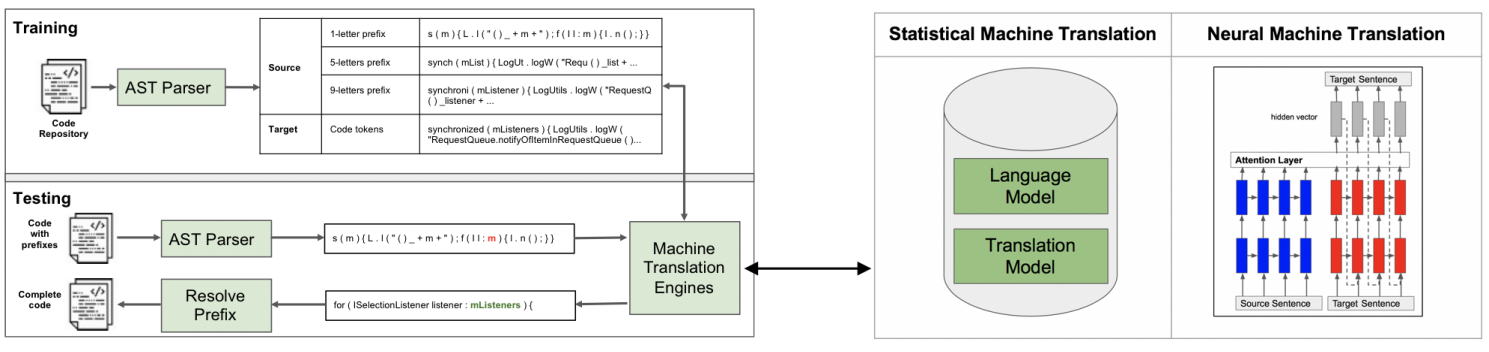

Figure 1: PrefixMap Architecture Overview

Language Model. In SMT, the LM is calculated for the target language, means the sequence of code tokens in our problem [8]. Recently, there are newer LM models that encoded neural network as mentioned in [22]. In SMT, it used the statistical language model approach called $n$-gram. The $n$-gram language model will assign probability for code sequences of the whole MethodDeclaration along with the sequence of code tokens inside the body of method. In general, the most useful purpose of LM is to calculate the probability of the last word of n-gram sequence given the previous code tokens. The LM approach is also applied in building code suggestion tool from very large code database such as [3] .

Theoretically, to predict the next code tokens, the larger number of $n$-gram produces the better of code token suggestion. However, large n-gram will cause exponential time increasing in the performance. In practical, Phrasal restricts the maximum size of $n$-gram as 7 . The fastest $n$-gram, uni-gram, is not usually used since I provide the estimation only by the number of appearance for that code tokens. The intuition of calculating the $\mathrm{n}$-gram for code tokens is estimating the probability of the current $n$ word given $n-1$ words by calculating the ratio between the number of appearance of sequence of $n$ words per the number of appearance in sequence of $\mathrm{n}$ words. Smoothing is the technique that the LM are required if a word appeared in the unseen context to avoid the LM to assign zero probability. In our SMT implementation, we use the Kneser-Ney smoothing method, which is proposed by KenLM $[8,16,17]$. We have the probabilistic model $P_{L M}$ as follow:

$$
\begin{aligned}
& P_{L M}\left(\operatorname{code}_{n} \mid \operatorname{code}_{1}^{n-1}\right)= \\
& \left.\quad u\left(\operatorname{code}_{n} \mid \operatorname{code}_{1}^{n-1}\right)+b\left(\operatorname{code}_{1}^{n-1}\right) * P_{L M}\left(\operatorname{code}_{n} \mid \operatorname{code}_{2}^{n-1}\right)\right)
\end{aligned}
$$

In Formula 1, the language model probability for the $n^{\text {th }}$ code token is calculated recursively by the respected probability of the $(n)^{t h}$ token given (n-2) tokens. This probability has 2 other elements for normalization, called pseudo probability $\mathrm{u}(\mathrm{)}$ ) and backoff metric $b$ [17]. The recursion will stop at the unigram distribution in Formula 2. In this Formula, [] is the empty sequence. the code tokens which are unseen in data will have the probability of the second operand, since $u()$ is equals to Zero.Example of how LM represented in PrefixMap in line 5 example 1 is shown in Table 2.

$$
\left.P_{L M}\left(\operatorname{code}_{n} \mid[]\right)=u\left(\operatorname{code}_{n} \mid[]\right)+b(\epsilon) * \frac{1}{\mid \text { vocOfTokens } \mid}\right)
$$

In this example, we see that the output of $P_{L M}$ suggests the list of variables given 5 previous tokens in the 6-grams LM. It shows the most popular popular variable as the list variable which is the first candidate suggested by the LM. However, the inference between
Table 2: Example of 6-grams LM probability in Listing 1

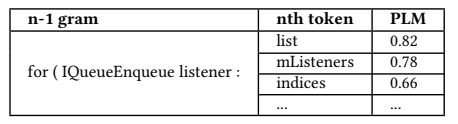

source and target language also depends on another probability along with the probability of the target language. We have the second element of the SMT, the translation model.

Translation Model. The translation model integrates the information from LM, the mapping probability of the prefixes given the code tokens (called the phrase translation probability) and the reordering score between translated phrase and input phrase. The final output of this step provided by Phrasal is a data structure called Phrase Translation Table, which contains probability for each phrase as candidate for translation. There are three stages in producing the phrase table. The first stage is word alignment, which is done by IBM model 1 by default [26]. Next, the phrase pairs of source and target languages are extracted. Final, the scoring phrase pairs process is done to assign the probability to phrase table. The probability of translation from prefixes to code tokens can be provided as follow:

Codes $_{\text {best }}=\operatorname{argmax}_{\text {codes }} p($ Prefixes $\mid$ Codes $) * p_{L M}($ Codes $)$

In Formula 3, the phrase translation probability $p$ (Prefixes|Codes) contains information about the association between source and target tokens and the probability for reordering the phrases order between source and target language. The association between the phrase of source given the phrase of target language can be scored by relative frequency of their co-occurrence in the training set [26]. There are several ways for estimating the phrase translation probability. In newest version of Phrasal, it used the Log Linear method, which calculate the translation model by Formula 4:

$$
p(x)=\exp \left(\sum_{i=1}^{3} \lambda_{i} * h_{i}(x)\right)
$$

In this formula, the final translation probability will depend on the exponential of three features functions and 3 hyper parameters. They are $\log (\theta)$ as the $\log$ arithm of relative frequency, $\log (d)$ as the $\log$ of reorder penalty, and $\log \left(P_{L M}\right)$ as the logarithm of language model probability. The input variable $\mathrm{x}$ is a random variable contains information about the candidate phrases and the position of phrases in source language. 
Table 3: Phrase table result of Line 5 Listing 1

\begin{tabular}{|c|l|l|}
\hline source & \multicolumn{1}{|c|}{ target } & $\mathbf{p}(\mathbf{t} \mid \mathbf{s})$ \\
\hline for $(I 1: \mathrm{m}$ & for (IQueueEnqueue listener $: \mathrm{mListeners}$ & 0.88 \\
\hline for $(\mathrm{I} 1: \mathrm{m}$ & for (Index listItem $: \mathrm{m}$ & 0.62 \\
\hline
\end{tabular}

The translation probability extracted by the phrase table after training the data of Line 5 in Listing 1 is shown in Table 3. In this table, we see that the probability for the phrase contained the sequence $f$ ( I 1 : returns the phrase that contains the correct translation result as $\mathrm{mListeners.}$

Optimization of SMT for PrefixMap. Since this corpus for PrefixMap has chracteristics of consistent length and consistent order between source and target language, we alternate the original SMT model for suit with our problem. First, we create our own alignment of source and target tokens instead of using IBM Model 1. Second, with the original output from SMT, we provide an algorithm to reverse the reordered phrases. These steps ensure the output of SMT always be consistent in length and order with the input prefixes.

\subsection{Neural Machine Translation for Prefix Map}

For NMT, we implement the solution for PrefixMap based on wellknown tool Google NMT [30]. The strength of NMT relies on the Encoder-Decoder architecture. As its name imply, the Encoder and Decoder layer provide an intermediate layers to convert the input sentence to a vector by an encoder model and convert from output vector to sequence of tokens in target language. These vector, called thought vector, can represent the meaning of sentence which capture other structures of sentences between source and target language. There are several details architecture of the Encode-Decoder model. Convolutional Neural Network $(\mathrm{CNN})$ was proposed to learn for image processing and unsupervised learning $[1,13]$. Graph Convolutional Network (GCN) is another architecture which can be used for graph structure data and semi-supervised classification [24]. Recurrent Neural Network (RNN) is the architecture used in sequence to sequence translation [42]. Long Short Term Memory (LSTM) is an improvement of RNN which help the training process to memorize the context efficiently [42]. Based on characteristics of PrefixMap, we select RNN model along with LSTM as recurrent unit and Attention mechanism to apply for this problem.

Recurrent Neural Network. Instead of splitting the sentence as prefixes by phrases, the RNN create a sequence of vectors represented for each prefixes and provide the translated output one by one. The information about the memory of translation will be represented as a hidden state vector $h_{i}$. Compared to n-gram which is usually be limited by the length of phrase, the hidden state vector can learn the information of previous prefixes at a very long distance. RNN provides a strategy to calculate the hidden state vector at time step $t$ based on the hidden state of previous time step by the following formula:

$$
\begin{gathered}
h_{t}=\sigma\left(W_{x h} x_{t}+W_{h h} h_{t-1}\right) \\
p_{t}=\operatorname{softmax}\left(W_{h y} h_{t}\right)
\end{gathered}
$$

In formula 5, the $\sigma$ function is the non linear functions such as signmoid and tanh. The probability over the set of candidate translation at time step $t$ will be calculated by formula 6 . In this formula, $x_{i}$ is

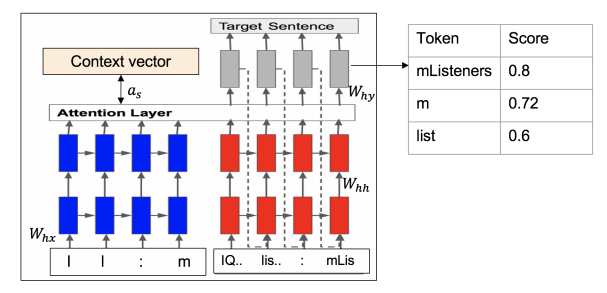

Figure 2: NMT translation result of Listing 1

an embedded vector representation for prefix $i$ in the sequence of source sentence. There are 3 weight matrices that can be learned from the training. They are the recurrent weight $W_{h h}$, the feedforward weight $W_{x h}$ and the output weight $W_{h y}$. The LSTM is selected as recurrent unit which contributes to layer of RNN.

Attention Mechanism. The attention model provide the better context embedding which allow to get more information from the source representation instead of used only at the initialization of hidden state. There are 2 types of attention, global attention which considers all previous prefixes and local attention which considers subset of input prefixes. In this research, we use the global attention as the information. The attention model creates connections between source hidden state and target hidden state. The heart of this problem is to design a context vector $c_{t}$. The new hidden state layers for target decoder can be estimated by the $\tanh ($ ) function:

$$
\bar{h}_{t}=\tanh \left(W_{c} \mid c_{t} ; h_{t}\right)
$$

To calculate the context vector, Google NMT propose an internal vector called variable length alignment weight vector $a_{t}$ which embeds the information of both source states and target states. Given the source state s, the alignment weight vector is calculated as formula 8 from [30]:

$$
a_{t}(s)=\frac{\exp \left(\operatorname{score}\left(h_{t}, \bar{h}_{s}\right)\right)}{\sum\left(\exp \left(\operatorname{score}\left(h_{t}, \bar{h}_{s^{\prime}}\right)\right)\right)}
$$

The score function in formula 8 can be calculated by dot or concatenation by vectors [30]. To see how RNN and Attention work, we can look at the illustration on Listing 1. In Figure 2, the step of translation is done by following steps. First, a sequence of input prefixes will be translated to a sequence of vectors $x_{1}, \ldots, x_{k}$. In the training of RNN, $W_{x h}$ represents for the relation between input vector and the hidden source, weight $W_{h h}$ propagates the relation between different hidden vector, while $W_{h y}$ represents the weight of output vector and hidden target state. The final score for each candidate of prefix $\mathrm{m}$ are shown in 2.

Optimization of NMT for PrefixMap. From formula 6, we see that the NMT provides a distribution for all posible output, mean all possible tokens in the vocabulary. So that, it cannot work with too large vocabulary with more than 40000 words [37]. To overcome this challenge, we replace the prefixes/ tokens with less than 10 times appearance as Unknown token. We apply the same algorithm to reverse the order of the output of NMT like SMT model.

\section{EVALUATION}

We implement the tool PrefixMap with 2 Machine Translation engines.Since the idea of PrefixMap can also be applicable in different 
types of documents, we provide our experiment from all 3 types of documentations. They are Natural Language, Software Documentation and Programming Language. We also discuss on our results on comparing between Neural Machine Translation versus Statistical Machine Translation on this problem. We target to have the information about the prefix resolution based on different types of code tokens. In general, we want to answer the following research questions:

(1) RQ1: Is prefix resolution important in programming language?

(2) RQ2: Does NMT outperform SMT on PrefixMap by NLP translation evaluation metrics?

(3) RQ3: Does NMT outperform SMT on PrefixMap by SE translation evaluation metrics?

(4) RQ4: How SMT and NMT perform with different types of code prefixes?

(5) RQ5: How SMT and NMT perform with ambiguous code prefixes?

\subsection{Metrics for MT Evaluation}

We use 2 metrics reflect the view point of NLP and SE: the BLEU score and the exact matching accuracy.

BLEU. Bilingual Evaluation Understudy score, called the BLEU score, is the fundamental metric for comparing the actual output and expected output of the MT problem [34]. The BLEU score will take input as pair of expected and translated result, it will output as the score from 0 to 1 to reflect the similarity at n-gram level of word. The higher of BLEU, the better of translation engine performed. This score is calculated based on the co-occurrence at of n-gram between expected and translated sentence along with strategies for penalty and smoothing [34]. We select the n-gram at 4-gram, and using the BLEU score implementation from Google NMT [30].

Exact Match Accuracy. In our MT problem, the requirement of good translation is not only restricted at the similar at n-gram. Similar to [37], another translation based approach in Software Engineering, we evaluate the Exact Match Accuracy at words level Given the ith index of source sequence pre $f i x_{i}$ and of the expected sequence expect $_{i}$ and of the translated sequence translate $_{i}$, we compare the match between the expect ${ }_{i}$ and translate $_{i}$. Since we have training data and testing data, we have Out of Vocabulary (OOV) cases. OOV has 2 types: Out of Source (OOS) means pre fix didn't appear in the training data and Out of Target (OOT) means expect $_{i}$ didn't exist in the training data. Since the prefix mapping process should suggest meaningful code token, we avoid evaluating the cases that the expected token was the same with prefix token.

Training and Test Sets. For each types of documentation, we split the data by $80 \%$ of training, $10 \%$ of validation and $10 \%$ of testing. The requirement of validation data is important in machine translation for tuning the training model to get optimized hyper parameters [30]. The selection of validation and testing is done randomly from the list of pairs of prefixes and tokens.

\subsection{Corpus Preparation}

We do the evaluation on 3 types of documentation:

Natural Language (NL). We collect all English sentences from the large scale corpus of English-German translation in NLP in [30].
Table 4: Configuration of Statistical Machine Translation Model

\begin{tabular}{|l|l|}
\hline Key & Value \\
\hline MAX_PHRASE_LEN & 7 \\
\hline Memory & $26 \mathrm{~GB}$ \\
\hline ttable-limit & 250 \\
\hline distortion-limit & 50 \\
\hline stack & 100 \\
\hline
\end{tabular}

This corpus contains 1,15 millions sentences.

Software Documentation (SD).We use the Conala corpus from [50]. This corpus contains Python software documentation as 116000 English sentences.

Programming Language (PL).We collect 1000 Java projects from MSR 2013 corpus [3]. We extract 560000 pairs of source and target tokens. The algorithm for extracting source and target language can be found in section 3. The application for PrefixMap in SD and PL is code completion for code like terms in SD and code tokens in PL. We also apply PrefixMap in popular corpus of NL for word completion, to reveal the differences of accuracy when applies PrefixMap in natural language and in programming language. For the variation of n-letter prefixes, we do the evaluation based on 3 levels: 1-letter prefix, 5-letters prefix and 9-letters prefix. To select these 3 types of prefix, we analysis the average length of each tokens for corpus, which brings the result on Table 6. It shows that the average length of code tokens is 18 letters, which means the developers need the auto code completion when they write 9-letters prefix or any prefix with less than 9 letters.

\subsection{MT Models Configurations}

To train SMT and NMT models, we use a high end computer with 32GB of RAM and an Nvidia RTX 2080 card with 8GB of GPU.

Statistical Machine Translation. We use the default configuration suggested by Phrasal [14].The details of configuration is shown in 4.

Neural Machine Translation. Number of units in each hidden layer affects the accuracy [40]. Increasing number of hidden unit can improve the accuracy but reduce the time performance since we need to change the batch size. So for NMT, we have 2 configurations as shown in Table 5.

\subsection{RQ1: Analysis on Length of Tokens in NL, SD and PL}

To answer RQ1, we analyze the average length of tokens in the target language of our NL, SD and PL corpus. The result is shown in Table 6. By this table, we show that the average length of words in NL is over 6 letters in English. This is consistent with our hypothesis since we consider that NL usually intend to describe a single word in a token. Besides, the size of vocabulary in in NL corpus is around 20000, which is feasible for NMT to train and get the result without removing any unknown tokens.

For the SD and PL corpus, the result shows different characteristics of these data. For the average length, the PL corpus gains highest length of letter per separate words at over 16. The PL also 
Table 5: Configuration of Neural Machine Translation Models

\begin{tabular}{|l|l|l|}
\hline Key & Config A (512 units) & Config B (1024 units) \\
\hline attention & normed_bahdanau & normed_bahdanau \\
\hline attention_architecture & gnmt_v2 & gntt_v2 \\
\hline batch_size & $\mathbf{3 2}$ & $\mathbf{1 6}$ \\
\hline beam_width & 10 & 10 \\
\hline decay_scheme & luong10 & luong10 \\
\hline dropout & 0.2 & 0.2 \\
\hline encoder_type & gnmt & gnmt \\
\hline infer_batch_size & 32 & $\mathbf{1 6}$ \\
\hline infer_mode & greedy & greedy \\
\hline init_op & uniform & uniform \\
\hline init_weight & 0.1 & 0.1 \\
\hline learning_rate & 1 & 1 \\
\hline length_penalty_weight & 1 & 1 \\
\hline num_decoder_layers & 2 & 2 \\
\hline num_encoder_layers & 2 & 2 \\
\hline num_train_steps & $\mathbf{3 4 0 0 0 0}$ & $\mathbf{6 8 0 0 0 0}$ \\
\hline num__units & $\mathbf{5 1 2}$ & $\mathbf{1 0 2 4}$ \\
\hline optimizer & sgd & sgd \\
\hline share_vocab & FALSE & FALSE \\
\hline src_max_len & 255 & 255 \\
\hline steps_per_stats & 100 & 100 \\
\hline & & \\
\hline
\end{tabular}

Table 6: Analysis on Length of Tokens in NL, SD and PL

\begin{tabular}{|l|l|l|}
\hline Target Languages & Vocab size & Average token length \\
\hline Natural Language & 19038 & 6.08 \\
\hline Software Documentation & 134886 & 11.77 \\
\hline Programming Language & 541275 & 18.38 \\
\hline
\end{tabular}

reveals a high average length of letters as over 11 . This is the expected result, since in SD and PL, sentences are usually made by developers. Unlike NL, developers have to mention about AST elements such as method names and class names, which increases the number of letters per each tokens. This means the code completion tool that allows getting code from prefix is needed.

The other points from Table 6 also shows that the vocabulary size of each corpus are varied. In contrast to NL, the SD and PL corpus contains a remarkably bigger vocabulary. In our assumption, this fact is due to 2 reasons. First, the PL and SD contains tokens that mentioned several words. It means that they can be combination of tuple or triple of words instead of uni-word per token like NL. Secondly, the SD and PL can be developed by many developers with different code naming style, which cause many rare words appeared in the corpus.

Answer of RQ1: Code tokens in Java usually have more number of letters than popular NL and SD corpus.

\subsection{RQ2: BLEU Score Evaluation for Prefix Mapping in NL, SD and PL}

In this experiment, we provide the translation for both NL, SD and PL corpus for SMT and configuration A of the SMT. The results are shown in Table 7 and Table 8. Along with these experiments, we have a verification experiment, which we run the configuration $\mathrm{A}$ on the English-German translation by [30]. We got the accuracy of BLEU score at $\mathbf{2 9 . 7 4}$ compared to 29.9 of Google NMT, which shows the validity of our NMT configurations. For NL SD, we use 1-letter prefix as the source language.

From Table 7, we show that the increasing of BLEU score from NL, SD to PL. The NL has the lowest BLEU score. The reason is
Table 7: BLEU Score Evaluation using SMT and NMT in NL, SD and PL

\begin{tabular}{|l|l|l|}
\hline \multicolumn{1}{|c|}{ Corpus } & SMT & NMT (config A) \\
\hline NL & 13.36 & 8.67 \\
\hline SD & 53.11 & 24.09 \\
\hline PL (1-letter) & 63.4 & 53.99 \\
\hline PL (5-letters) & 84.9 & 64.33 \\
\hline PL (9-letters) & 92.61 & 74.42 \\
\hline
\end{tabular}

Table 8: BLEU Score Comparison between Config A and B of NMT

\begin{tabular}{|l|l|l|}
\hline \multicolumn{1}{|c|}{ Corpus } & NMT (Config A) & NMT (Config B) \\
\hline PL (1-letter) & 53.99 & 52.78 \\
\hline PL (5-letters) & 64.33 & 73.39 \\
\hline PL (9-letters) & 74.42 & 79.12 \\
\hline
\end{tabular}

that we are doing the mapping from a context of 26 letters in NL to the target tokens which contains 19000 words, which caused challenges for the MT models. The PL corpus at 1-letter prefix returns surprisingly higher BLEU score. It shows the potential of capturing the code tokens based on code context can be better than in NL and SD. The BLEU score increases if we change the length of the prefixes.

We talk about the comparison between MT models. We got the accuracy of SMT outperforms the NMT with all of the corpus. This fact shows the strength of SMT to resolve the characteristic of consistent order between source and target language. We have a comparison of BLEU score between configuration A and B. It shows that the accuracy in configuration $B$ increases, which shows the important of increasing number of hidden units.

Answer of RQ2: SMT outperforms NMT significantly in accuracy of prefixes' translation measured by BLEU score.

\subsection{RQ3: Exact Match Accuracy Comparison of Prefix Mapping in NL, SD and PL}

The exact match accuracy are shown in Table 9 and Table 10. From these tables, we show the accuracy of the PrefixMap varied depending on the types of n-letters prefixes. We don't have the OOS cases in the NL, SD and PL with 1-letter prefix. For the SMT, we achieve over $65 \%$ of precision score, showing the strength of SMT in this type of problem. For 9-letter prefixes, the accuracy gained to $\mathbf{9 0 \%}$, means if developers wrote 9 letters of the code token, there are 9 per 10 cases the tool suggested correctly. We didn't include the non-useful suggestions counted to this accuracy. In the other words, the expected tokens need to be longer in letter than the prefixes.

For the NMT, the result is remarkably lower for config A but is improved in config B. We got the accuracy ranged from $61 \%$ from 1-letter prefix to $74 \%$ for 9-letters prefixes for config A of NMT. For config B, the precision ranked from $58.62 \%$ to $82.55 \%$. Though the gap between configuration B and SMT is only $8 \%$, another problem of the NMT is the OOV tokens. For 9-prefixes letter, we got the OOV of NMT as high as third times the OOV of SMT. This is caused by 
Table 9: Exact Match Accuracy Comparison between SMT and NMT Config A

\begin{tabular}{|l|l|l|l|l|l|l|l|l|}
\hline Corpus & \multicolumn{9}{|c|}{ SMT } \\
\hline & Correct & Incorrect & OOS & OOT & OOV & Prec & Rec & F1 \\
\hline NL & 12671 & 35890 & 0 & 196 & 196 & $26.09 \%$ & $98.48 \%$ & $41.25 \%$ \\
\hline SD & 40656 & 20176 & 0 & 1855 & 1855 & $66.83 \%$ & $95.64 \%$ & $78.68 \%$ \\
\hline PL (1-letter) & 53868 & 28529 & 0 & 4090 & 4090 & $65.38 \%$ & $92.94 \%$ & $76.76 \%$ \\
\hline PL (5-letters) & 40164 & 7897 & 540 & 3337 & 3877 & $83.57 \%$ & $91.20 \%$ & $87.22 \%$ \\
\hline PL (9-letters) & 20554 & 2207 & 1374 & 1753 & 3127 & $90.30 \%$ & $86.80 \%$ & $88.51 \%$ \\
\hline & \multicolumn{7}{|c|}{ NMT (Config A) } \\
\hline & Correct & Incorrect & OOS & OOT & OOV & Prec & Rec & F1 \\
\hline NL & 10487 & 38074 & 0 & 196 & 196 & $21.60 \%$ & $98.17 \%$ & $35.40 \%$ \\
\hline SD & 26237 & 31700 & 0 & 4750 & 4750 & $45.29 \%$ & $84.67 \%$ & $59.01 \%$ \\
\hline PL (1-letter) & 44510 & 28711 & 0 & 13266 & 13266 & $60.79 \%$ & $77.04 \%$ & $67.96 \%$ \\
\hline PL (5-letters) & 25565 & 13895 & 3758 & 8720 & 12478 & $64.79 \%$ & $67.20 \%$ & $65.97 \%$ \\
\hline PL (9-letters) & 11778 & 4217 & 7263 & 2630 & 9893 & $73.64 \%$ & $54.35 \%$ & $62.54 \%$ \\
\hline
\end{tabular}

Table 10: Exact Match Accuracy Comparison of NMT model Config B

\begin{tabular}{|c|l|l|l|l|l|l|l|l|}
\hline Corpuss & \multicolumn{9}{|c|}{ NMT (Config B) } \\
\hline & Correct & Incorrect & OOS & OOT & OOV & Precision & Recall & F1 \\
\hline PL (1-letter) & 42919 & 30302 & 0 & 1326 & 13266 & $58.62 \%$ & $76.39 \%$ & $66.33 \%$ \\
\hline PL (5-letters) & 30853 & 8607 & 3758 & 8720 & 12478 & $78.19 \%$ & $71.20 \%$ & $74.53 \%$ \\
\hline PL (9-letters) & 13204 & 2791 & 7263 & 2630 & 9893 & $82.55 \%$ & $57.17 \%$ & $67.55 \%$ \\
\hline
\end{tabular}

the fact that there is a set of words required to change to Unknown words, which can badly impact the total accuracy of NMT.

Answer of RQ3: SMT outperforms NMT significantly in accuracy of prefixes' translation measured by F1 score.

\subsection{RQ4: Analysis on Types of Tokens in Naming Conventions for Prefixes}

To setup this experiment, we provide a module that check the regular expression of each tokens in the testing data set. There are regular expressions on checking if a numeric, a class name, a variable, a method name or a string literal. We found and add the regular expressions for checking at well-known online resource as $[39,43]$. Considering the Configuration B as a higher accuracy NMT model compared to configuration A. The results are shown in Table 11 and Table 12.

The result shows the consistent between NMT and SMT configuration. The two types of token received lowest accuracy is Numeric and String literal. The numeric tokens got precision as $34 \%$ in 1-letter prefix of SMT and got $24.71 \%$ in 1-letter prefix of NMT configuration B. The String literal got $28 \%$ in each configuration. This fact reveals the challenge of code suggestion for numeric and string literal, since the they are not only depend on the tokens but also depend on the control flow or data flow graph of the program. For main types of tokens, we got the highest accuracy for class name while the variable name and constanct tokens achieved the equal results in both SMT and NMT. It is explainable since the good class names can be reused popular by developers which helps the SMT model to learn the prediction. Method name, in the other hands, varied based on the purpose of each developers.

Answer of RQ4: For prefixes' translation, numeric and string literal caused challenges for get good accuracy while class name achieved highest accuracy.
Table 11: Analysis Result on types of tokens for PrefixMap by SMT

\begin{tabular}{|l|l|l|l|l|l|l|l|l|}
\hline \multicolumn{8}{|c|}{ SMT (1-letter prefix) } \\
\hline Type of Tokens & Cor... & Incor... & OOS & OOT & OOV & Prec & Rec & F1 \\
\hline Total: & 53868 & 28529 & 0 & 4090 & 4090 & $65.38 \%$ & $92.94 \%$ & $76.76 \%$ \\
\hline NumericType: & 163 & 304 & 0 & 28 & 28 & $34.90 \%$ & $85.34 \%$ & $49.54 \%$ \\
\hline ClassNameType: & 6154 & 2793 & 0 & 317 & 317 & $68.78 \%$ & $95.10 \%$ & $79.83 \%$ \\
\hline VariableType: & 26937 & 14888 & 0 & 1503 & 1503 & $64.40 \%$ & $94.72 \%$ & $76.67 \%$ \\
\hline MethodNameType: & 12908 & 6695 & 0 & 501 & 501 & $65.85 \%$ & $96.26 \%$ & $78.20 \%$ \\
\hline StringLiteralType: & 628 & 1585 & 0 & 841 & 841 & $28.38 \%$ & $42.75 \%$ & $34.11 \%$ \\
\hline ConstanctType: & 1321 & 821 & 0 & 281 & 281 & $61.67 \%$ & $82.46 \%$ & $70.57 \%$ \\
\hline OtherType: & 5757 & 1443 & 0 & 619 & 619 & $79.96 \%$ & $90.29 \%$ & $84.81 \%$ \\
\hline \multicolumn{7}{|c|}{ SMT (5-letters prefix) } \\
\hline Total: & 40164 & 7897 & 540 & 3337 & 3877 & $83.57 \%$ & $91.20 \%$ & $87.22 \%$ \\
\hline NumericType: & 81 & 65 & 7 & 12 & 19 & $55.48 \%$ & $81.00 \%$ & $65.85 \%$ \\
\hline ClassNameType: & 6986 & 755 & 40 & 270 & 310 & $90.25 \%$ & $95.75 \%$ & $92.92 \%$ \\
\hline VariableType: & 17271 & 3861 & 258 & 1096 & 1354 & $81.73 \%$ & $92.73 \%$ & $86.88 \%$ \\
\hline MethodNameType: & 10924 & 2057 & 23 & 474 & 497 & $84.15 \%$ & $95.65 \%$ & $89.53 \%$ \\
\hline StringLiteralType: & 1219 & 389 & 100 & 730 & 830 & $75.81 \%$ & $59.49 \%$ & $66.67 \%$ \\
\hline ConstanctType: & 1182 & 404 & 29 & 243 & 272 & $74.53 \%$ & $81.29 \%$ & $77.76 \%$ \\
\hline OtherType: & 2501 & 366 & 83 & 512 & 595 & $87.23 \%$ & $80.78 \%$ & $83.88 \%$ \\
\hline & \multicolumn{8}{|c|}{ SMT (9-letters prefix) } \\
\hline Total: & 20554 & 2207 & 1374 & 1753 & 3127 & $90.30 \%$ & $86.80 \%$ & $88.51 \%$ \\
\hline NumericType: & 11 & 3 & 2 & 0 & 2 & $78.57 \%$ & $84.62 \%$ & $81.48 \%$ \\
\hline ClassNameType: & 4279 & 233 & 116 & 160 & 276 & $94.84 \%$ & $93.94 \%$ & $94.39 \%$ \\
\hline VariableType: & 6436 & 950 & 511 & 431 & 942 & $87.14 \%$ & $87.23 \%$ & $87.19 \%$ \\
\hline MethodNameType: & 6963 & 557 & 185 & 279 & 464 & $92.59 \%$ & $93.75 \%$ & $93.17 \%$ \\
\hline StringLiteralType: & 868 & 179 & 288 & 456 & 744 & $82.90 \%$ & $53.85 \%$ & $65.29 \%$ \\
\hline ConstanctType: & 923 & 155 & 104 & 114 & 218 & $85.62 \%$ & $80.89 \%$ & $83.19 \%$ \\
\hline OtherType: & 1074 & 130 & 168 & 313 & 481 & $89.20 \%$ & $69.07 \%$ & $77.85 \%$ \\
\hline
\end{tabular}

Table 12: Analysis Result on types of tokens for PrefixMap by NMT

\begin{tabular}{|l|l|l|l|l|l|l|l|l|}
\hline \multicolumn{7}{|c|}{ NMT in Config B (1-letter prefix) } \\
\hline Type of Tokens & Cor... & Incor... & OOS & OOT & OOV & Prec & Rec & F1 \\
\hline Total: & 42919 & 30302 & 0 & 13266 & 13266 & $58.62 \%$ & $76.39 \%$ & $66.33 \%$ \\
\hline NumericType: & 86 & 262 & 0 & 147 & 147 & $24.71 \%$ & $36.91 \%$ & $29.60 \%$ \\
\hline ClassNameType: & 4554 & 3343 & 0 & 1367 & 1367 & $57.67 \%$ & $76.91 \%$ & $65.91 \%$ \\
\hline VariableType: & 21774 & 16366 & 0 & 5188 & 5188 & $57.09 \%$ & $80.76 \%$ & $66.89 \%$ \\
\hline MethodNameType: & 10742 & 7065 & 0 & 2297 & 2297 & $60.32 \%$ & $82.38 \%$ & $69.65 \%$ \\
\hline StringLiteralType: & 354 & 902 & 0 & 1798 & 1798 & $28.18 \%$ & $16.45 \%$ & $20.77 \%$ \\
\hline ConstanctType: & 875 & 586 & 0 & 962 & 962 & $59.89 \%$ & $47.63 \%$ & $53.06 \%$ \\
\hline OtherType: & 4534 & 1778 & 0 & 1507 & 1507 & $71.83 \%$ & $75.05 \%$ & $73.41 \%$ \\
\hline \multicolumn{7}{|c|}{ NMT in Config B (5-letters prefix) } \\
\hline Total: & 30853 & 8607 & 3758 & 8720 & 12478 & $78.19 \%$ & $71.20 \%$ & $74.53 \%$ \\
\hline NumericType: & 40 & 18 & 84 & 23 & 107 & $68.97 \%$ & $27.21 \%$ & $39.02 \%$ \\
\hline ClassNameType: & 5473 & 1245 & 444 & 889 & 1333 & $81.47 \%$ & $80.41 \%$ & $80.94 \%$ \\
\hline VariableType: & 13575 & 4153 & 1402 & 3356 & 4758 & $76.57 \%$ & $74.05 \%$ & $75.29 \%$ \\
\hline MethodNameType: & 8843 & 2366 & 227 & 2042 & 2269 & $78.89 \%$ & $79.58 \%$ & $79.23 \%$ \\
\hline StringLiteralType: & 512 & 202 & 862 & 862 & 1724 & $71.71 \%$ & $22.90 \%$ & $34.71 \%$ \\
\hline ConstanctType: & 727 & 223 & 305 & 603 & 908 & $76.53 \%$ & $44.46 \%$ & $56.25 \%$ \\
\hline OtherType: & 1683 & 400 & 434 & 945 & 1379 & $80.80 \%$ & $54.96 \%$ & $65.42 \%$ \\
\hline \multicolumn{7}{|c|}{$\mathbf{N M T}$ in Config B (9-letters prefix) } \\
\hline Total: & 13204 & 2791 & 7263 & 2630 & 9893 & $82.55 \%$ & $57.17 \%$ & $67.55 \%$ \\
\hline NumericType: & 6 & 3 & 6 & 1 & 7 & $66.67 \%$ & $46.15 \%$ & $54.55 \%$ \\
\hline ClassNameType: & 3082 & 580 & 827 & 299 & 1126 & $84.16 \%$ & $73.24 \%$ & $78.32 \%$ \\
\hline VariableType: & 3632 & 1244 & 2645 & 807 & 3452 & $74.49 \%$ & $51.27 \%$ & $60.74 \%$ \\
\hline MethodNameType: & 5254 & 673 & 1335 & 722 & 2057 & $88.65 \%$ & $71.86 \%$ & $79.38 \%$ \\
\hline StringLiteralType: & 251 & 75 & 1168 & 297 & 1465 & $76.99 \%$ & $14.63 \%$ & $24.58 \%$ \\
\hline ConstanctType: & 456 & 91 & 632 & 117 & 749 & $83.36 \%$ & $37.84 \%$ & $52.05 \%$ \\
\hline OtherType: & 523 & 125 & 650 & 387 & 1037 & $80.71 \%$ & $33.53 \%$ & $47.37 \%$ \\
\hline
\end{tabular}

\subsection{RQ5: Analysis on the Accuracy of PrefixMap on Ambiguous Tokens}

In the last experiment, we analyze whether the number of prefixes to code token mappings can affect the accuracy. For each prefix in the training set, we run a program to check how many distinct code tokens are mapped to that prefix in the target language. The prefix that has more mapped tokens can be considered as more ambiguous. The result can be shown in Table 13 for SMT and Table 14 for NMT configuration B. The first observation we got is that in 1-letter prefix, there is no case of mapping 1-1 to tokens in both SMT and NMT. This fact is explainable since the vocabulary of the source language contains only letters in alphabet and number digits, which are less than 100 prefixes. Besides, there are a large percentage of the prefixes has more than 100 mappings with 1letter prefix corpus. For the 1-letter prefix, the SMT got accuracy 
Table 13: Analysis Result on how PrefixMap can handle Ambiguous tokens by SMT

\begin{tabular}{|l|l|l|l|l|l|l|}
\hline \multicolumn{7}{|c|}{ SMT (1-letter prefix) } \\
\hline NumofMap & $\mathbf{1}$ & $\mathbf{2 - 1 0}$ & $\mathbf{1 1 - 2 0}$ & $\mathbf{2 1 - 5 0}$ & $\mathbf{5 1 - 1 0 0}$ & $\mathbf{> 1 0 0}$ \\
\hline & Precision & Precision & Precision & Precision & Precision & Precision \\
\hline NumericType: & $0.00 \%$ & $0.00 \%$ & $0.00 \%$ & $0.00 \%$ & $0.00 \%$ & $34.90 \%$ \\
\hline ClassNameType: & $0.00 \%$ & $0.00 \%$ & $0.00 \%$ & $0.00 \%$ & $0.00 \%$ & $68.78 \%$ \\
\hline VariableType: & $0.00 \%$ & $0.00 \%$ & $0.00 \%$ & $100.00 \%$ & $0.00 \%$ & $64.40 \%$ \\
\hline MethodNameType: & $0.00 \%$ & $0.00 \%$ & $0.00 \%$ & $0.00 \%$ & $0.00 \%$ & $65.85 \%$ \\
\hline StringLiteralType: & $0.00 \%$ & $0.00 \%$ & $0.00 \%$ & $0.00 \%$ & $0.00 \%$ & $28.38 \%$ \\
\hline ConstanctType: & $0.00 \%$ & $0.00 \%$ & $0.00 \%$ & $0.00 \%$ & $0.00 \%$ & $61.67 \%$ \\
\hline OtherType: & $0.00 \%$ & $40.00 \%$ & $97.35 \%$ & $83.18 \%$ & $0.00 \%$ & $70.68 \%$ \\
\hline Total of tokens: & 0 & 10 & 2416 & 221 & 1 & 79749 \\
\hline Percentage: & $0.00 \%$ & $0.01 \%$ & $2.93 \%$ & $0.27 \%$ & $0.00 \%$ & $96.79 \%$ \\
\hline \multicolumn{7}{|c|}{ SMT (5-letter prefix) } \\
\hline NumericType: & $100.00 \%$ & $57.78 \%$ & $71.43 \%$ & $36.84 \%$ & $60.00 \%$ & $0.00 \%$ \\
\hline ClassNameType: & $100.00 \%$ & $91.83 \%$ & $88.90 \%$ & $87.06 \%$ & $90.34 \%$ & $89.09 \%$ \\
\hline VariableType: & $100.00 \%$ & $80.09 \%$ & $78.40 \%$ & $77.36 \%$ & $82.91 \%$ & $81.70 \%$ \\
\hline MethodNameType: & $100.00 \%$ & $94.60 \%$ & $89.77 \%$ & $90.62 \%$ & $89.03 \%$ & $80.35 \%$ \\
\hline StringLiteralType: & $100.00 \%$ & $85.04 \%$ & $75.63 \%$ & $74.70 \%$ & $68.45 \%$ & $65.50 \%$ \\
\hline ConstanctType: & $100.00 \%$ & $83.66 \%$ & $79.35 \%$ & $75.09 \%$ & $66.67 \%$ & $58.60 \%$ \\
\hline OtherType: & $100.00 \%$ & $87.09 \%$ & $84.58 \%$ & $90.91 \%$ & $91.85 \%$ & $82.38 \%$ \\
\hline Total of tokens: & 1980 & 6425 & 3375 & 6411 & 7017 & 22853 \\
\hline Percentage: & $4.12 \%$ & $13.37 \%$ & $7.02 \%$ & $13.34 \%$ & $14.60 \%$ & $47.55 \%$ \\
\hline \multicolumn{7}{|c|}{ SMT (9-letter prefix) } \\
\hline NumericType: & $100.00 \%$ & $100.00 \%$ & $50.00 \%$ & $75.00 \%$ & $0.00 \%$ & $0.00 \%$ \\
\hline ClassNameType: & $100.00 \%$ & $94.22 \%$ & $90.73 \%$ & $89.12 \%$ & $89.56 \%$ & $83.10 \%$ \\
\hline VariableType: & $100.00 \%$ & $84.45 \%$ & $77.86 \%$ & $72.23 \%$ & $74.80 \%$ & $57.25 \%$ \\
\hline MethodNameType: & $100.00 \%$ & $92.64 \%$ & $87.54 \%$ & $91.82 \%$ & $85.10 \%$ & $80.07 \%$ \\
\hline StringLiteralType: & $100.00 \%$ & $84.38 \%$ & $69.66 \%$ & $68.29 \%$ & $71.74 \%$ & $59.85 \%$ \\
\hline ConstanctType: & $100.00 \%$ & $80.74 \%$ & $66.27 \%$ & $72.46 \%$ & $64.71 \%$ & $66.67 \%$ \\
\hline OtherType: & $100.00 \%$ & $88.58 \%$ & $81.52 \%$ & $90.79 \%$ & $88.46 \%$ & $53.33 \%$ \\
\hline Total of tokens: & 6360 & 9952 & 1808 & 2550 & 1302 & 789 \\
\hline Percentage: & $27.94 \%$ & $43.72 \%$ & $7.94 \%$ & $11.20 \%$ & $5.72 \%$ & $3.47 \%$ \\
\hline
\end{tabular}

about $70 \%$ while the NMT got $59 \%$ for very ambiguous tokens. For 5-letters and 9-letter prefixes, the accuracy of SMT decreases from unambiguous tokens to very ambiguous tokens. In the NMT with 9-letters prefix and for very ambiguous tokens, these tokens are considered as Unknown due to their rarely appeared in the data set, cause the NMT to decrease. In general, the SMT outperforms the NMT for almost all of accuracy experiments.

Answer of RQ5: For the translation of most ambiguous tokens, the SMT outperforms NMT in both 3 types of prefix. In NMT, sparsity tokens with more than 9 letters are usually be converted to Unknown tokens which caused low accuracy for this MT engine.

\section{RELATED WORK}

The characteristics of our parallel corpus appeared in other SE problems $[18,37]$. In these works, SMT outperforms NMT in accuracy. In general, SE researches have specific characteristics of corpus, which brings rooms for deep learning and machine learning to improve their approaches. Other researches show drawbacks of Machine Learning in SE. [21] and point out the drawbacks of machine learning approach for method name recommendation that is usually suggest too simple method names. [41] shows that the original SMT has problems of exponential time increasing with big data. For the code suggestion area, other research works focuses on a specific types of code tokens. [49] suggested method name based on Hierachical Attention Networks, and [5] suggested method name and class name. In our work, we intend to generate all types of tokens based on writing the abbreviations or prefixes.
Table 14: Analysis Result on how PrefixMap can handle Ambiguous tokens by NMT

\begin{tabular}{|l|l|l|l|l|l|l|}
\hline \multicolumn{7}{|c|}{ NMT in Config B (1-letter prefix) } \\
\hline NumofMap & $\mathbf{1}$ & $\mathbf{2 - 1 0}$ & $\mathbf{1 1 - 2 0}$ & $\mathbf{2 1 - 5 0}$ & $\mathbf{5 1 - 1 0 0}$ & $>\mathbf{1 0 0}$ \\
\hline & Precision & Precision & Precision & Precision & Precision & Precision \\
\hline NumericType: & $0.00 \%$ & $0.00 \%$ & $66.67 \%$ & $29.79 \%$ & $30.77 \%$ & $23.16 \%$ \\
\hline ClassNameType: & $0.00 \%$ & $0.00 \%$ & $25.00 \%$ & $62.50 \%$ & $0.00 \%$ & $57.68 \%$ \\
\hline VariableType: & $0.00 \%$ & $0.00 \%$ & $0.00 \%$ & $0.00 \%$ & $19.64 \%$ & $57.15 \%$ \\
\hline MethodNameType: & $0.00 \%$ & $0.00 \%$ & $0.00 \%$ & $0.00 \%$ & $50.00 \%$ & $60.33 \%$ \\
\hline StringLiteralType: & $0.00 \%$ & $0.00 \%$ & $0.00 \%$ & $0.00 \%$ & $0.00 \%$ & $28.18 \%$ \\
\hline ConstanctType: & $0.00 \%$ & $0.00 \%$ & $66.67 \%$ & $0.00 \%$ & $0.00 \%$ & $59.96 \%$ \\
\hline OtherType: & $0.00 \%$ & $89.25 \%$ & $50.00 \%$ & $66.29 \%$ & $35.29 \%$ & $58.76 \%$ \\
\hline Total: & 0 & 2661 & 32 & 324 & 90 & 70114 \\
\hline Percentage: & $0.00 \%$ & $3.63 \%$ & $0.04 \%$ & $0.44 \%$ & $0.12 \%$ & $95.76 \%$ \\
\hline \multicolumn{7}{|c|}{$\mathbf{N M T}$ in Config B (5-letters prefix) } \\
\hline NumericType: & $76.47 \%$ & $66.67 \%$ & $64.29 \%$ & $0.00 \%$ & $0.00 \%$ & $0.00 \%$ \\
\hline ClassNameType: & $79.67 \%$ & $84.24 \%$ & $79.10 \%$ & $76.19 \%$ & $84.81 \%$ & $0.00 \%$ \\
\hline VariableType: & $70.64 \%$ & $74.03 \%$ & $78.03 \%$ & $85.35 \%$ & $64.47 \%$ & $57.97 \%$ \\
\hline MethodNameType: & $91.36 \%$ & $88.80 \%$ & $82.14 \%$ & $81.87 \%$ & $74.68 \%$ & $63.74 \%$ \\
\hline StringLiteralType: & $75.74 \%$ & $67.24 \%$ & $77.88 \%$ & $70.83 \%$ & $0.00 \%$ & $0.00 \%$ \\
\hline ConstanctType: & $87.64 \%$ & $76.84 \%$ & $75.58 \%$ & $47.73 \%$ & $53.42 \%$ & $0.00 \%$ \\
\hline OtherType: & $92.73 \%$ & $75.98 \%$ & $73.26 \%$ & $88.68 \%$ & $77.80 \%$ & $83.33 \%$ \\
\hline Total: & 3324 & 10580 & 6002 & 10938 & 5283 & 3333 \\
\hline Percentage: & $8.42 \%$ & $26.81 \%$ & $15.21 \%$ & $27.72 \%$ & $13.39 \%$ & $8.45 \%$ \\
\hline \multicolumn{7}{|c|}{$\mathbf{N M T}$ in Config B (9-letters prefix) } \\
\hline NumericType: & $0.00 \%$ & $66.67 \%$ & $0.00 \%$ & $0.00 \%$ & $0.00 \%$ & $0.00 \%$ \\
\hline ClassNameType: & $83.72 \%$ & $86.38 \%$ & $76.40 \%$ & $68.18 \%$ & $0.00 \%$ & $0.00 \%$ \\
\hline VariableType: & $77.08 \%$ & $72.33 \%$ & $70.32 \%$ & $68.24 \%$ & $0.00 \%$ & $0.00 \%$ \\
\hline MethodNameType: & $92.88 \%$ & $88.57 \%$ & $80.41 \%$ & $67.58 \%$ & $77.50 \%$ & $0.00 \%$ \\
\hline StringLiteralType: & $78.17 \%$ & $71.43 \%$ & $87.10 \%$ & $0.00 \%$ & $0.00 \%$ & $0.00 \%$ \\
\hline ConstanctType: & $88.08 \%$ & $73.86 \%$ & $50.00 \%$ & $0.00 \%$ & $0.00 \%$ & $0.00 \%$ \\
\hline OtherType: & $73.49 \%$ & $78.28 \%$ & $91.72 \%$ & $66.67 \%$ & $0.00 \%$ & $0.00 \%$ \\
\hline Total: & 7403 & 6748 & 1300 & 504 & 40 & 0 \\
\hline Percentage: & $46.28 \%$ & $42.19 \%$ & $8.13 \%$ & $3.15 \%$ & $0.25 \%$ & $0.00 \%$ \\
\hline
\end{tabular}

\section{CONCLUSION}

In this work, we propose PrefixMap, a code suggestion tool for all types of code tokens in Java programming language. To realize our idea, we propose two Machine Translation models, Statistical Machine Translation and Neural Machine Translation, which learn the information from source language as the space of abbreviation or prefix to the target language as actual code tokens. Our work shows that we got an accuracy from $60 \%$ to $90 \%$ for SMT and from $59 \%$ to $83 \%$ for NMT. In the Machine Learning point of view, we reveal a class of parallel corpus which SMT can learn more information and get better accuracy on NMT in Software Engineering. Two of the characteristics of SE parallel corpus are the unknown tokens problem and being consistent on the length and the order of the source and target corpus. While the accuracy on different prefixes shows how SMT outperforms NMT, these characteristics provide initial take-away messages about the reasons of why NMT results low accuracy for this problem.

There are few limitations of our work. First, we only select 3 types of prefixes for our evaluation. We will extend our work to evaluate the accuracy of inference based on a range of lengths for prefixes. Secondly, we use an adhoc approach to treat the unknown token for NMT which is based on removing sparsity tokens from the corpus. In future work, we will study how abbreviations are written by developers to support more types of abbreviation suggestions instead of suggesting only by prefix, and apply optimization of NMT in other area such as [46] to improve the accuracy. The artifact of PrefixMap is available at [38]. 


\section{REFERENCES}

[1] Hamed Habibi Aghdam et al. 2017. Guide to Convolutional Neural Networks: A Practical Application to Traffic-Sign Detection and Classification (1st ed.). Springer Publishing Company, Incorporated.

[2] A. Alhefdhi et al. 2018. Generating Pseudo-Code from Source Code Using Deep Learning. In 2018 25th Australasian Software Engineering Conference (ASWEC) 21-25. https://doi.org/10.1109/ASWEC.2018.00011

[3] M. Allamanis et al. 2013. Mining source code repositories at massive scale using language modeling. In 2013 10th Working Conference on Mining Software Repositories (MSR). 207-216. https://doi.org/10.1109/MSR.2013.6624029

[4] Miltiadis Allamanis et al. 2015. Bimodal Modelling of Source Code and Natural Language. In Proceedings of the 32nd International Conference on International Conference on Machine Learning - Volume 37 (Lille, France) (ICML'15). JMLR.org, 2123-2132.

[5] Miltiadis Allamanis et al. 2015. Suggesting Accurate Method and Class Names. In Proceedings of the 2015 10th foint Meeting on Foundations of Software Engineering (Bergamo, Italy) (ESEC/FSE 2015). Association for Computing Machinery, New York, NY, USA, 38-49. https://doi.org/10.1145/2786805.2786849

[6] Miltiadis Allamanis et al. 2018. A Survey of Machine Learning for Big Code and Naturalness. ACM Comput. Surv. 51, 4, Article 81 (July 2018), 37 pages. https://doi.org/10.1145/3212695

[7] Project Android. 2019. Example of method fireQueueStateChanged() in the Android project. https://tinyurl.com/ukgnrph.

[8] Stanley F. Chen et al. 1996. An Empirical Study of Smoothing Techniques for Language Modeling. In Proceedings of the 34th Annual Meeting on Association for Computational Linguistics (Santa Cruz, California) (ACL '96). Association for Computational Linguistics, USA, 310-318. https://doi.org/10.3115/981863.981904

[9] Marta R. Costa-Jussà et al. 2014. Statistical Machine Translation Enhancements through Linguistic Levels: A Survey. ACM Comput. Surv. 46, 3, Article 42 (Jan. 2014), 28 pages. https://doi.org/10.1145/2518130

[10] Hoa Khanh Dam et al. 2016. A deep language model for software code. CoRR abs/1608.02715 (2016). arXiv:1608.02715 http://arxiv.org/abs/1608.02715

[11] Mehdi Drissi et al. 2018. Program Language Translation Using a Grammar Driven Tree-to-Tree Model. CoRR abs/1807.01784 (2018). arXiv:1807.01784 http://arxiv.org/abs/1807.01784

[12] Fabio Ferreira et al. 2019. Software Engineering Meets Deep Learning: A Literature Review. arXiv:1909.11436 [cs.SE]

[13] Torumoy Ghoshal et al. 2019. Improving Performance of Convolutional Neural Networks via Feature Embedding. In Proceedings of the 2019 ACM Southeast Conference (Kennesaw, GA, USA) (ACM SE '19). Association for Computing Machinery, New York, NY, USA, 31-38. https://doi.org/10.1145/3299815.3314429

[14] Spence Green et al. 2014. Phrasal: A Toolkit for New Directions in Statistical Machine Translation. In In Proceddings of the Ninth Workshop on Statistical Machine Translation.

[15] Xiaodong Gu et al. 2016. Deep API Learning. In Proceedings of the 2016 24th ACM SIGSOFT International Symposium on Foundations of Software Engineering (Seattle, WA, USA) (FSE 2016). Association for Computing Machinery, New York, NY, USA, 631-642. https://doi.org/10.1145/2950290.2950334

[16] Kenneth Heafield. 2011. KenLM: Faster and Smaller Language Model Queries. In Proceedings of the EMNLP 2011 Sixth Workshop on Statistical Machine Translation Edinburgh, Scotland, United Kingdom, 187-197. https://kheafield.com/papers/ avenue/kenlm.pdf

[17] Kenneth Heafield et al. 2013. Scalable Modified Kneser-Ney Language Model Estimation. In Proceedings of the 51st Annual Meeting of the Association for Computational Linguistics (Volume 2: Short Papers). Association for Computational Linguistics, Sofia, Bulgaria, 690-696. https://www.aclweb.org/anthology/P13-2121

[18] Vincent J. Hellendoorn et al. 2018. Deep Learning Type Inference. In Proceedings of the 2018 26th ACM Joint Meeting on European Software Engineering Conference and Symposium on the Foundations of Software Engineering (Lake Buena Vista, FL, USA) (ESEC/FSE 2018). Association for Computing Machinery, New York, NY, USA, 152-162. https://doi.org/10.1145/3236024.3236051

[19] Abram Hindle et al. 2012. On the Naturalness of Software. In Proceedings of the 34th ICSE 2012 (Zurich, Switzerland) (ICSE '12). IEEE Press, 837-847.

[20] Eclipse JDT. 2020. Eclipse JDT. https://tinyurl.com/yx5hto3f.

[21] L. Jiang et al. 2019. Machine Learning Based Recommendation of Method Names: How Far are We. In 2019 34th IEEE/ACM International Conference on Automated Software Engineering (ASE). 602-614.

[22] Kun Jing et al. 2019. A Survey on Neural Network Language Models. CoRR abs/1906.03591 (2019). arXiv:1906.03591 http://arxiv.org/abs/1906.03591

[23] Aggarwal K et al. 2015. Using machine translation for converting Python 2 to Python 3 code. arXiv:3:e1459v1 https://peerj.com/preprints/1459v1/

[24] Thomas N. Kipf et al. 2016. Semi-Supervised Classification with Graph Convolutional Networks. CoRR abs/1609.02907 (2016). arXiv:1609.02907 http: //arxiv.org/abs/1609.02907

[25] Guillaume Klein et al. 2017. OpenNMT: Open-Source Toolkit for Neural Machine Translation. In Proc. ACL. https://doi.org/10.18653/v1/P17-4012
[26] Philipp Koehn. 2010. Statistical Machine Translation (1st ed.). Cambridge University Press, USA.

[27] Xiaochen Li et al. 2018. Deep Learning in Software Engineering. arXiv:1805.04825 [cs.SE]

[28] Xi Victoria Lin et al. 2018. NL2Bash: A corpus and semantic parser for natural language interface to the Linux operating system. In LREC: Language Resources and Evaluation Conference. Miyazaki, Japan.

[29] Zhongxin Liu et al. 2018. Neural-Machine-Translation-Based Commit Message Generation: How Far Are We? In Proceedings of the 33rd ACM/IEEE International Conference on Automated Software Engineering (Montpellier, France) (ASE 2018). Association for Computing Machinery, New York, NY, USA, 373-384. https: //doi.org/10.1145/3238147.3238190

[30] Minh-Thang Luong et al. 2017. Neural Machine Translation (seq2seq) Tutorial. https://github.com/tensorflow/nmt (2017).

[31] Thang Luong et al. 2014. Addressing the Rare Word Problem in Neural Machine Translation. CoRR abs/1410.8206 (2014). arXiv:1410.8206 http://arxiv.org/abs/ 1410.8206

[32] Anh Tuan Nguyen and others. 2014. Migrating Code with Statistical Machine Translation. In Companion Proceedings of the 36th ICSE (Hyderabad, India) (ICSE Companion 2014). Association for Computing Machinery, New York, NY, USA, 544-547. https://doi.org/10.1145/2591062.2591072

[33] Y. Oda et al. 2015. Learning to Generate Pseudo-Code from Source Code Using Statistical Machine Translation (T). In Automated Software Engineering (ASE) 2015. 574-584. https://doi.org/10.1109/ASE.2015.36

[34] Kishore Papineni et al. 2002. BLEU: A Method for Automatic Evaluation of Machine Translation. In Proceedings of the 40th Annual Meeting on Association for Computational Linguistics (Philadelphia, Pennsylvania) (ACL '02). Association for Computational Linguistics, USA, 311-318. https://doi.org/10.3115/1073083. 1073135

[35] Thai-Hoang Pham et al. 2017. On the Use of Machine Translation-Based Approaches for Vietnamese Diacritic Restoration. CoRR abs/1709.07104 (2017). arXiv:1709.07104 http://arxiv.org/abs/1709.07104

[36] H. Phan et al. 2017. Statistical Learning for Inference between Implementations and Documentation. In 2017 IEEE/ACM 39th International Conference on Software Engineering (ICSE). IEEE Computer Society, Los Alamitos, CA, USA, 27-30. https: //doi.org/10.1109/ICSE-NIER.2017.9

[37] Hung Phan et al. 2018. Statistical Learning of API Fully Qualified Names in Code Snippets of Online Forums. In Proceedings of the 40th International Conference on Software Engineering (Gothenburg, Sweden) (ICSE '18). Association for Computing Machinery, New York, NY, USA, 632-642. https://doi.org/10.1145/3180155. 3180230

[38] PrefixMap. 2020. PrefixMap data. https://pdhung3012.github.io/prefixmap.html.

[39] RegexTester. 2019. Regular Expression collection site 2. https://www.regextester. com/97778.

[40] ResearchGate. 2019. Question of Hidden Unit in NMT. https://tinyurl.com/ j5b2o3r.

[41] C. M. K. Saifullah et al. 2019. Learning from Examples to Find Fully Qualified Names of API Elements in Code Snippets. In 2019 34th IEEE/ACM International Conference on Automated Software Engineering (ASE). 243-254. https://doi.org/ 10.1109/ASE.2019.00032

[42] Alex Sherstinsky. 2018. Fundamentals of Recurrent Neural Network (RNN) and Long Short-Term Memory (LSTM) Network. CoRR abs/1808.03314 (2018). arXiv:1808.03314 http://arxiv.org/abs/1808.03314

[43] StackOverflow. 2019. Regular Expression collection site 1. https://stackoverflow. $\mathrm{com} /$.

[44] James V. Stone. 2013. Bayes' Rule: A Tutorial Introduction to Bayesian Analysis. Sebtel Press.

[45] Thanh Van Nguyen et al. 2016. Characterizing API Elements in Software Documentation with Vector Representation. In Proceedings of the 38th International Conference on Software Engineering Companion (Austin, Texas) (ICSE '16). Association for Computing Machinery, New York, NY, USA, 749-751. https://doi.org/10.1145/2889160.2892658

[46] Xing Wang et al. 2016. Neural Machine Translation Advised by Statistical Machine Translation. CoRR abs/1610.05150 (2016). arXiv:1610.05150 http://arxiv.org/abs/ 1610.05150

[47] Xinyi Wang et al. 2018. A Tree-based Decoder for Neural Machine Translation. CoRR abs/1808.09374 (2018). arXiv:1808.09374 http://arxiv.org/abs/1808.09374

[48] Yonghui Wu et al. 2016. Google's Neural Machine Translation System: Bridging the Gap between Human and Machine Translation. CoRR abs/1609.08144 (2016). http://arxiv.org/abs/1609.08144

[49] Sihan Xu et al. 2019. Method Name Suggestion with Hierarchical Attention Networks. In Proceedings of the 2019 ACM SIGPLAN Workshop on Partial Evaluation and Program Manipulation (Cascais, Portugal) (PEPM 2019). Association for Computing Machinery, New York, NY, USA, 10-21. https://doi.org/10.1145/ 3294032.3294079

[50] Pengcheng Yin et al. 2018. Learning to Mine Aligned Code and Natural Language Pairs from Stack Overflow. In International Conference on Mining Software Repositories (MSR). ACM, 476-486. https://doi.org/10.1145/3196398.3196408 